\title{
Phase Retrieval in Terahertz Time-Domain Measurements: a "how to" Tutorial
}

\author{
Jepsen, Peter Uhd
}

Published in:

Journal of Infrared, Millimeter and Terahertz Waves

Link to article, DOI:

10.1007/s10762-019-00578-0

Publication date:

2019

Document Version

Peer reviewed version

Link back to DTU Orbit

Citation $(A P A)$ :

Jepsen, P. U. (2019). Phase Retrieval in Terahertz Time-Domain Measurements: a "how to" Tutorial. Journal of Infrared, Millimeter and Terahertz Waves, 40(4), 395-411. https://doi.org/10.1007/s10762-019-00578-0

\section{General rights}

Copyright and moral rights for the publications made accessible in the public portal are retained by the authors and/or other copyright owners and it is a condition of accessing publications that users recognise and abide by the legal requirements associated with these rights.

- Users may download and print one copy of any publication from the public portal for the purpose of private study or research.

- You may not further distribute the material or use it for any profit-making activity or commercial gain

- You may freely distribute the URL identifying the publication in the public portal

If you believe that this document breaches copyright please contact us providing details, and we will remove access to the work immediately and investigate your claim. 


\title{
Phase retrieval in terahertz time-domain measurements: A "how to" tutorial
}

\author{
Peter Uhd Jepsen \\ Department of Photonics Engineering, Technical University of Denmark, DK-2800 Kongens Lyngby, Denmark \\ Email:puje@fotonik.dtu.dk, Phone:(+45) 45255711
}

\begin{abstract}
:
Terahertz time-domain spectroscopy (THz-TDS) is in many ways a well-established, proven and versatile spectroscopic technique that is frequently and routinely used in many laboratories. The basis of high-quality optical data on materials using THz-TDS is the correct extraction of the complex-valued dielectric properties (index of refraction, permittivity or conductivity) from the recorded amplitude and phase of the involved $\mathrm{THz}$ signals. The focus of this paper is to discuss stable methods for finding the physically meaningful frequency-dependent optical phase from time-domain signals, thereby avoiding some of the unphysical solutions to the inversion problem that is the central part of THz-TDS analysis. The paper discusses problems associated with the positioning of the THz signal in the recorded time window, phase offsets due to noise in the experimental data, and phase correction in the case of strongly dispersive media such as transparent semiconductors in the frequency range below but close to the transverse optical phonons.
\end{abstract}




\section{Introduction}

Terahertz time-domain spectroscopy (THz-TDS) is a well-established spectroscopic technique that since 1989 has been used in almost 6,500 peer-reviewed scientific works with over 100,000 citations ${ }^{1}$. The very first THz-TDS demonstration by van Exter, Fattinger, and Grischkowsky [1] already demonstrated the basic and unique feature of THz-TDS, namely that the coherent generation and detection of an ultrashort THz pulse can be applied for a highly versatile spectroscopy technique, where the frequency-resolved amplitude and phase of the detected signal are recovered directly and without any model-based assumptions about the signal. The extraction of dielectric properties (index of refraction and absorption coefficient) from THz-TDS measurements relies heavily on correct retrieval of the optical phase of the THz wave. The general review of THz-TDS measurement and analysis by Withayachumnankul and Naftaly [2] discusses the fundamentals of phase retrieval in THz-TDS. In this paper, I will discuss some common pitfalls associated with a correct and physically meaningful treatment of the phase in a THz-TDS measurement, including correct estimation of general phase offsets and identification of situations where a standard blind phase unwrapping leads to incorrect estimation of the refractive index of a sample. Such situations occur for instance in highly dispersive samples, such as transparent polar semiconductors at frequencies below the fundamental transverse optical (TO) phonon.

There is a tremendous variety of applications of THz-TDS systems. While commercial systems are becoming more and more accessible, the majority of systems in use are custom-built by the same persons that operate them for spectroscopic applications. There are no universally established measurement protocols that ensure traceable results, and a recent study from the National Physical Laboratory shows a surprisingly large variation in spectroscopic results obtained on the same samples characterized by an international range of THz spectroscopy groups [3]. That important study indicated that as the THz-TDS technique now matures, the community shares a large responsibility, and needs to work together to establish and share common standards and best practices for recording and treatment of THz-TDS data. This tutorial contributes to the understanding of the optical phase in THz-TDS measurements, and advises methods that avoid common pitfalls in data analysis, thereby adding to the literature on best practices in THz-TDS [4-10]. While some of the discussed issues are trivial and others are more intricate and difficult to identify, the ambiguities associated with phase retrieval can play a role in the variability seen in results from different laboratories on the same materials.

The phase-sensitive detection is often emphasized as a unique capability of THz-TDS, but it should be emphasized that this is also possible with dispersive Fourier Transform Spectroscopy (DFTS), using incoherent light sources [11] and within an almost identical mathematical framework as is used in THz-TDS. DFTS was first demonstrated by Chamberlain, Gibbs, and Gebbie in 1963 [12], who used this technique for the measurement of the refractive index of crystalline quartz between 20 and $55 \mathrm{~cm}^{-1}(0.6-1.65 \mathrm{THz})$. The seminal THz-TDS article from Grischkowsky et al. from 1990 on the optical properties of dielectrics and semiconductors [13] applied THz-TDS to samples that had already been characterized by DFTS. THz-TDS demonstrated superior spectroscopic data quality combined with a straightforward data analysis and an optical setup that laboratories with access to femtosecond laser technology could build with standard components, thereby establishing THz-TDS as the technique of choice for future optical characterization in the far infrared. Today, the main use of DFTS technology is in optical coherence tomography (OCT), which is currently expanding its useful spectral range from the traditional near-infrared to the mid-infrared [14], and shares many characteristics with THz time-domain imaging

\footnotetext{
${ }^{1}$ Web of Science January 2019, search term “Terahertz AND time-domain”, excluding conference proceedings.
} 
(THz-TDI) $[15,16]$. Also scattering-type scanning near field microscopy (s-SNOM) has recently been combined with DFTS to enable phase-sensitive spectroscopy across the THz and mid-infrared with resolution of a few tens of nanometers [17], and recently, the same technique has been applied to nanoscale optical pump - mid-IR probe imaging with femtosecond time resolution [18]. Hence, retrieval of the optical phase in time-domain measurements is relevant for a very wide community in optics.

In a THz-TDS measurement, the raw data from the experiment are two time traces of the detected electric field, recorded under some reference condition $\left[E_{r e f}(t)\right]$ and after interaction (reflection or transmission) with the sample under investigation $\left[E_{\text {sam }}(t)\right]$. Spectroscopy is performed by Fourier transformation of the two time traces, $E_{r e f}(f)$ and $E_{\text {sam }}(f)$, where $f$ is the frequency. The amplitude and phase of the ratio of the two signals in frequency space (the complex-valued transmission function) is compared to a model of the transmission through the sample. Inversion of this relation is then performed in order to determine the best estimate of the frequency-dependent optical properties (complex index of refraction, permittivity or conductivity). In the general case, this inversion is a problem on its own that is outside the scope of this paper, and we refer to the existing literature on this, for instance the papers by Duvillaret et al. [4, 5], Pupeza et al. [6], Scheller et al. [7], and Kruger et al. [8]. In these and most other works, the THz beam is assumed a plane wave so that the sample is not altering the geometric propagation of the beam. However, if the THz beam is focused tightly onto the sample, significant errors in the optical parameter extraction can arise from the plane-wave approximation since the finite thickness of the sample, compared to the Rayleigh range of the THz focus, may alter the subsequent propagation and focusing of the broadband THz signal onto the detector. This effect was noted by Kužel et al. [19], who demonstrated a method that compensates the effect.

Here we will discuss some hidden pitfalls in the correct extraction of the refractive index from the measured phase. The discussion will be limited to the simple case of single-pass transmission THz-TDS, where echoes due to multiple reflections of the $\mathrm{THz}$ beam in the sample can be removed with simple windowing techniques. In this case, the measured transmission function is related to the index of refraction $n$ and absorption coefficient $\alpha$ by the simple relation [20]

$$
\tilde{T}(f)=|T| \exp (i \Delta \phi)=\frac{4 n}{(n+1)^{2}} \exp (-\alpha d / 2) \exp (2 \pi i(n-1) f d / c)
$$

Here $c$ is the speed of light in vacuum, and $d$ is the sample thickness. This expression is valid within the spectral range of the spectrometer, where the signal of both reference and sample spectra are above the noise floor of the system [10]. The optical constants are thus related to the transmission amplitude $|T|$ and phase difference $\Delta \phi$ as

$$
\begin{aligned}
& n(f)=1+\frac{c \Delta \phi}{2 \pi f d} \\
& \alpha(f)=-\frac{2}{d} \ln \left(\frac{(n+1)^{2}}{4 n}|T|\right) .
\end{aligned}
$$

Equations (1) and (2) are approximations to any experiment. The most important assumptions are as follows. The THz beam is treated as a plane wave at normal incidence (finite size of the THz beam is ignored). Alterations of the beam path due to the sample are ignored (i.e. the sample is thin compared to the Rayleigh range). Only the directly transmitted part of the signal is considered (no echoes in the sample due to multiple reflections). In more complete descriptions of the 
interaction between $\mathrm{THz}$ waves and a sample, Eqs. (1) and (2) are replaced with more complicated expressions that may or may not have closed-form analytical solutions. However, the substance of the discussion that follows remains valid also in such situations.

In transmission measurements, the optical phase of the beam transmitted through a sample of thickness $d$, compared to that of transmission through the same distance of air, directly determines the refractive index of the sample, as described by Eqs. (1) and (2),

$$
\Delta \phi=\left(k-k_{0}\right) d=\frac{2 \pi(n-1) f d}{c} .
$$

This means, that for a constant index of refraction, the phase difference increases monotonously with frequency. On the other hand, in a reflection measurement the phase of the detected signal after reflection from a sample, relative to the reflection from a reference surface such as metal $(r=-1)$ contains information about the phase of the reflection coefficient of the sample, and thus mainly gives information about the extinction coefficient of the material,

$$
\frac{\tilde{r}_{\text {sample }}}{r_{\text {reference }}}=-\tilde{r}_{\text {sample }}=|r| \exp (i \Delta \phi)=\frac{\tilde{n}-1}{\tilde{n}+1},
$$

where $\tilde{n}(f)=n+i \kappa=n+i \alpha c /(4 \pi f)$. In this case, the detected phase will be restricted to the $[-\pi ; \pi]$ range, and therefore the pitfalls related to correct phase retrieval described here are not as relevant as in transmission spectroscopy.

\section{THz-TDS phase retrieval}

From a mathematical point of view, the phase is defined as the arctangent of the ratio of the imaginary to the real part of the complex-valued ratio of the sample and reference signals. In other words, for a given complex-valued signal $A \exp (i \phi)=a+i b$ the phase is recovered as $\phi=\arctan (b / a)$. The arctangent function by definition returns angles only in the interval $[-\pi / 2 ; \pi / 2]$ (red curve, Figure 1). The four-quadrant arctangent function returns angles in the full $[-\pi ; \pi]$ range (orange curve, Figure 1). In either case, the continuous optical phase increase described by Eq. (3) (blue curve, Figure 1) is not fully recovered; the winding number in the complex plane is not maintained in the angle restoration. This leads to undesirable phase jumps that can, however, be corrected. This is typically done computationally in a loop where phase jumps between adjacent frequency points are tested; if larger than some threshold (typically $\pm \pi$ ) then all phase values above the given frequency are correspondingly offset by $\mp 2 \pi$. This very general and well-known process is referred to as "phase unwrapping". 


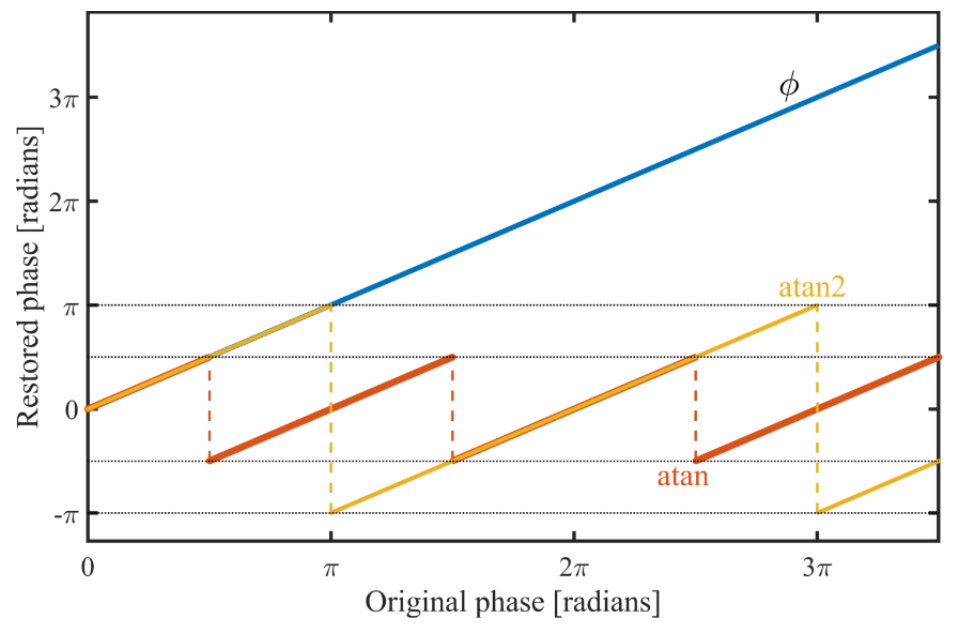

Figure 1. A continuous phase of a complex quantity (blue curve) can only be partially reconstructed by the arctangent function (atan, red curve) or the four-quadrant arctangent function (atan2, orange curve).

In the following we assume that the THz time traces are recorded at $N$ equidistant sample points with a step size $\Delta t$ and a total scan length $T=N \Delta t$. This results in a frequency resolution $\Delta f=1 / T$ and a Nyquist frequency $f_{\max }=1 /(2 \Delta t)$. Figure 2 shows an example of a THz-TDS measurement on a sample of quartz glass, thickness $d=1.067 \mathrm{~mm}$. The data sets consist of $N=346$ time points, spaced equidistantly by $\Delta t=0.0781 \mathrm{ps}$. This data set will be used in the following discussion. Figure 2(a) shows the time domain traces with indication of the arrival time of the absolute maximum of the signals. Figure 2(b) shows the amplitude of the Fourier transforms of the signals, and the gray area (also used on subsequent figures) indicates the high-frequency region where the signal approaches the noise floor of the experiment [10].
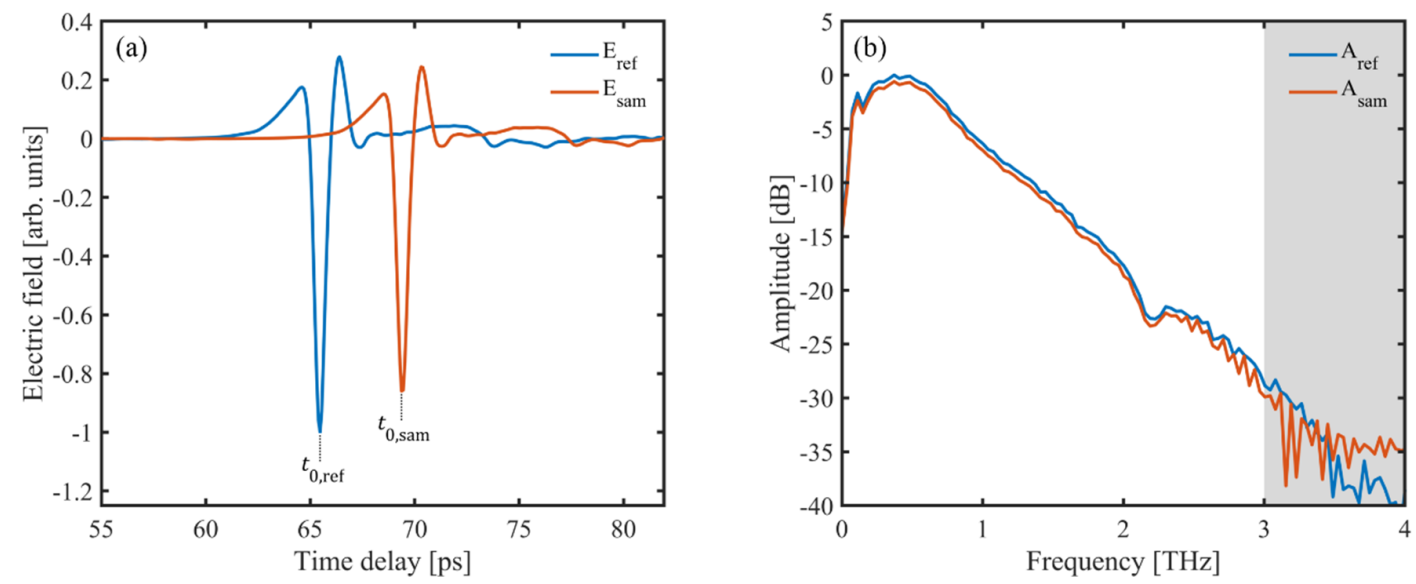

Figure 2. (a) Raw time-domain data for a THz-TDS measurement. The blue reference pulse is propagated through dry air and the red sample pulse is propagated through $1.067 \mathrm{~mm}$ quartz glass. (b) The frequency spectra of the two pulses. The gray-shaded region indicates the upper limit of useful spectral content of the signals.

Phase unwrapping can, under standard THz-TDS conditions, easily correct the undesired " $2 \pi$ phase jumps". However, the phase unwrapping works under the assumption that the regular phase increase between adjacent frequency points in the discrete Fourier transform of the THz signals is actually smaller than the threshold value for phase jump correction. 
Three common situations can challenge this assumption leading to more or less obvious mistakes in the evaluation of the refractive index.

For completeness, the standard method for phase unwrapping with a computer program is shown in Figure 3. The example shows an implementation in simple Matlab scripting language, and is portable to other programming languages with minimal modifications. The code works on an array phase of real numbers, of length N. Most mathematics software packages for scientific use, including Matlab, Python and Octave, feature built-in unwrapping functions.

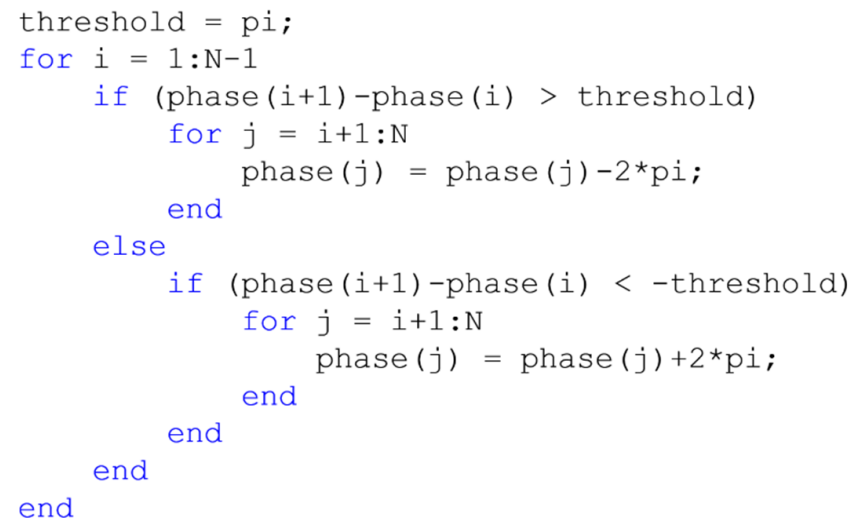

Figure 3. Phase unwrapping code, implemented in simple Matlab scripting.

Firstly, let us consider the general position of the THz pulse in the time window. If the position of the peak of the THz signal is at time $t_{0}\left(t_{0, \text { ref }}, t_{0, \text { sam }}\right.$, respectively, in Figure 2$)$ then the phase of its Fourier transform will be approximately

$$
\begin{aligned}
& \phi_{0, \text { ref }}(f)=2 \pi f t_{0, \text { ref }}, \\
& \phi_{0, \text { sam }}(f)=2 \pi f t_{0, \text { sam }} .
\end{aligned}
$$

This means that the phase jump between adjacent frequency points for any of the two linear phase functions in Eq. (5) will be

$$
\phi_{k}-\phi_{k-1}=2 \pi \Delta f t_{0}=2 \pi t_{0} / T
$$

which is larger than $\pi$ for $t_{0}>T / 2$. Hence, unwrapping of the phase with a threshold of $\pi$ will mistakenly overcorrect the phase curve, and lead to an apparent phase curve with negative slope. In that case, the unwrapped phase can be corrected by

$$
\phi^{\prime}=\phi+2 \pi f / \Delta f
$$

The same correction can be done automatically by subtracting the overall phase of the signals [Eq. (5)] before unwrapping, and then adding the same phase after unwrapping. This reduced phase is best calculated by multiplying the complex-valued spectra by $\exp \left(-i \phi_{0, \text { ref }}\right)$ and $\exp \left(-i \phi_{0, \text { sam }}\right)$, respectively, before unwrapping. 
In the following, the term "blind unwrapping" refers to the use of the algorithm in Figure 3 without further considerations, whereas the term "informed unwrapping" will refer to an unwrapping procedure where the noise properties of the data and the details of the sample are considered in the unwrapping procedure.

Figure 4 shows different representations of the frequency-dependent phases of the Fourier transforms in Figure 2. Figure 4(a) shows the raw phase angles, without unwrapping. Figure 4(b) shows the blindly unwrapped phases (blue and red curves) together with the expected phases (gray dotted and dashed curves) for the reference and sample signals, respectively, calculated from the peak positions in the time domain [Eq. (5)]. Figure 4(c) shows the phase when unwrapping is performed on the reduced phase, again compared to the overall phases expected from the delay of the signals. Finally, Figure 4(d) shows the phase difference (blue curve) used for calculation of the refractive index together with the overall linear phase difference (dotted, gray curve) between the signals. With the inclusion of the overall phase of the signals in the unwrapping procedure, it can be seen that unwrapping now works as intended. Since the subtraction of the overall phases of the signals lead to rather small variations of the phase spectrum, this method is very stable and can be implemented in an automated fashion in data analysis software.
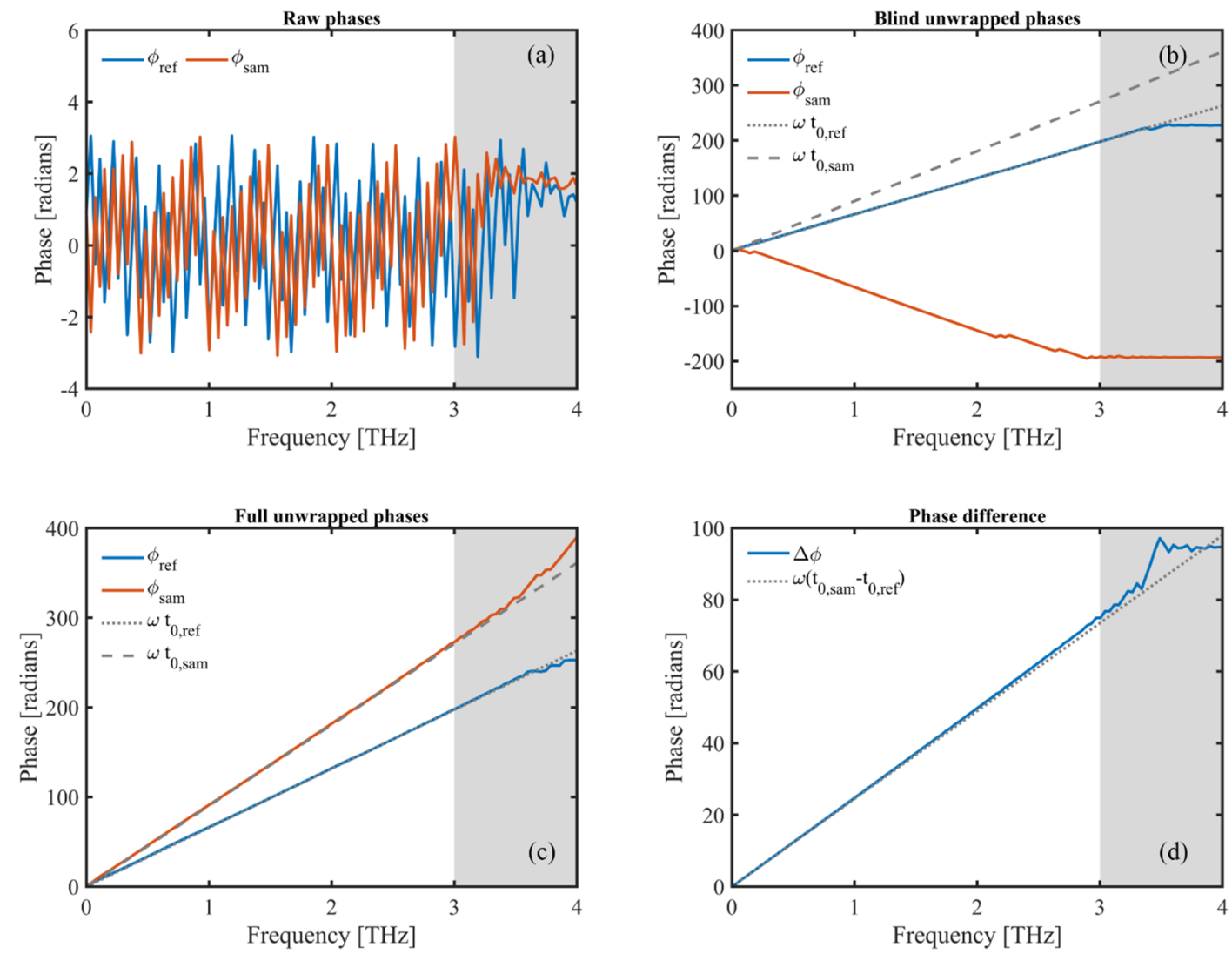

Figure 4: Phase information in THz-TDS. (a) Raw phase data retrieved from the Fourier transform of the two time traces in Figure 2. (b) Phases after blind unwrapping, with indication of the expected phase due to the temporal offset of each pulse (gray curves). (c) Correctly unwrapped phases, using Eq. (7), and (d) the final phase difference used to calculate the refractive index of the sample. 
Secondly, if the unwrapping takes place in spectral regions with low signal strength (at very low or very high frequencies, where typical THz-TDS signals are weak, or in spectral regions with very strong absorption of the sample), then at least one of the reference and sample signals is dominated by instrumental noise, and the phase information is scrambled. In this case, the calculated index of refraction is ill defined, as the phase difference will in practice be offset by multiples of $2 \pi$ due to phase unwrapping on too noisy phases. The optical phase should extrapolate to zero at low frequencies, so if there is a phase offset of $m \cdot 2 \pi$, then the extracted refractive index from a transmission measurement will diverge at low frequencies to

$$
n^{\prime}=n+m \cdot 2 \pi \frac{c}{\omega d},
$$

with a tell-tale $\pm \omega^{-1}$ low-frequency divergence. The effect of such accidental phase offset is shown in Figure 5(a). Since the refractive index is needed to account for reflection losses, the extracted absorption coefficient is also influenced by such phase offsets, as shown in Figure 5(b). Since the general phase curve deviates only weakly from the general linear behavior in most situations, the phase offset can be identified and compensated in an automatic manner by fitting a straight line to the phase data in the region of highest signal-to-noise ratio and extrapolating the linear fit to zero frequency. The extrapolated phase should be close to zero, and otherwise the phase curve can be shifted by a suitable multiple of $2 \pi$.
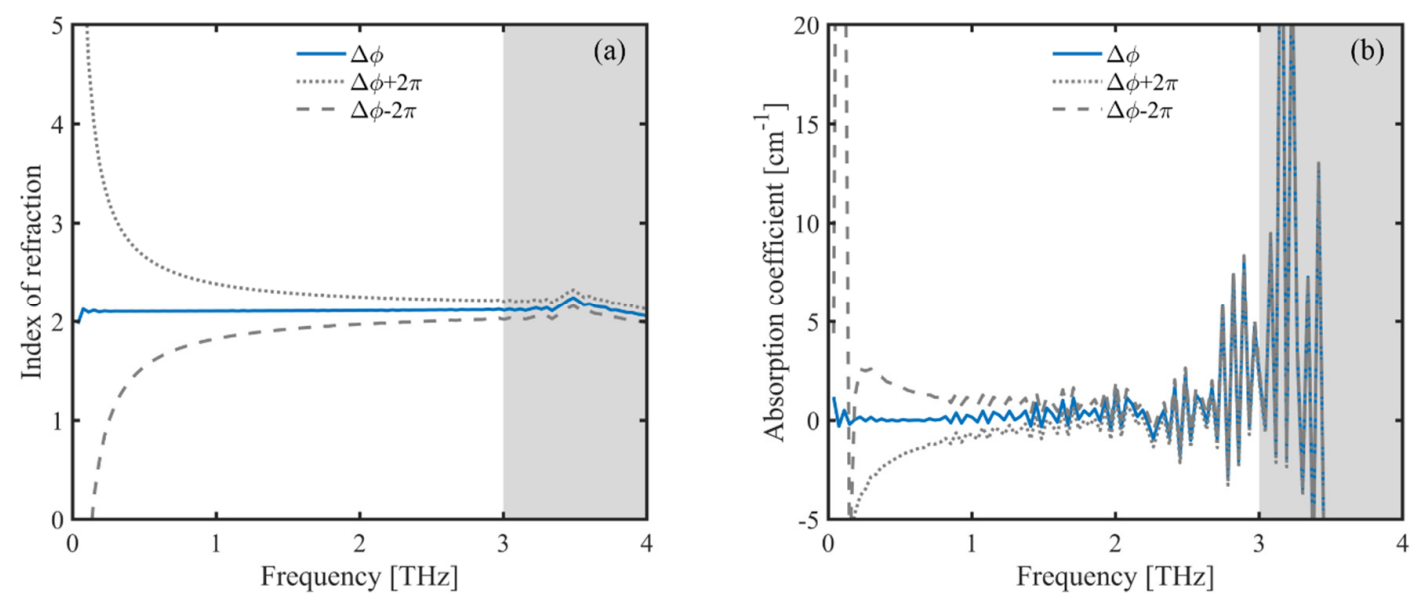

Figure 5: (a) The calculated refractive index (Eq. (2)) of the 1.067-mm thick quartz sample, based on the phase difference shown in Figure 4. An accidental phase offset of $\pm 2 \pi$ results in divergent refractive index, as indicated by the gray dashed and dotted curves. (b) The extracted absorption relies on the refractive index, hence such phase offset also influences the apparent absorption coefficient.

To summarize the previous discussion, the strategy for the most stable phase unwrapping is therefore to ensure that the slope of the phase to be unwrapped is as low as possible, in order to minimize the possibility of unintentional overcompensation of the phase. The following steps ensure this, by noting that the average slope of the phases of the reference and sample signals (Eq. (5)) can be subtracted from the raw phase of the Fourier transforms of the signals, and then added to the phase difference of the two signals after unwrapping. 
1) Locate the temporal position of the maximum of the absolute value of the reference and sample $\mathrm{THz}$ pulses, labeled $t_{0, r e f}$ and $t_{0, \text { sam }}$, respectively, and determine the temporal offset between the sample and reference time windows, $t_{\text {offset }}$.

2) Calculate the Fourier transforms of the reference and sample signals, $\tilde{E}_{\text {ref }}(\omega)$ and $\tilde{E}_{\text {sam }}(\omega)$.

3) Calculate the reduced phase of the reference and sample signals,

$$
\begin{aligned}
& \phi_{\text {ref }}^{\text {red }}=\angle\left(\tilde{E}_{r e f}(\omega) \exp \left(-i \phi_{0, r e f}\right)\right), \\
& \phi_{\text {sam }}^{\text {red }}=\angle\left(\tilde{E}_{\text {sam }}(\omega) \exp \left(-i \phi_{0, \text { sam }}\right)\right) .
\end{aligned}
$$

where $\angle$ denotes the phase of a complex number.

4) Perform a standard unwrap of the reduced phase differences,

$$
\Delta \phi_{0}^{*}=\operatorname{unwrap}\left(\phi_{s a m}^{\text {red }}-\phi_{r e f}^{\text {red }}\right) .
$$

5) Check if there is a global phase offset (integer multiples of $2 \pi$ ) by linear regression - fit a linear function $\phi(\omega)=A \omega+B$ to the central part of the phase curve (in the spectral range of highest dynamic range). Offset the phase difference by an accordingly,

$$
\Delta \phi_{0}=\Delta \phi_{0}^{*}-2 \pi \cdot \operatorname{int}(B / 2 \pi)
$$

Where int refers to the integer number closest to $B / 2 \pi$.

6) Find the full phase difference between the sample and reference signals as

$$
\Delta \phi=\Delta \phi_{0}-\phi_{0, r e f}+\phi_{0, \text { sam }}+\phi_{o f f s e t}
$$

The third case of phase problems is less obvious than the two situations described above. If the sample is thick (in the sense that there is a large difference in arrival times of the reference and sample pulses) and the frequency resolution in the experiment is limited, then the condition of small phase increase between adjacent frequency points may not be met by the experimental conditions, and simple phase unwrapping will no longer work as intended.

\section{Phase unwrapping in dispersive media}

If the starting point of the time window for recording of the sample signal is the same as that of the reference signal, the phase of the sample signal with sample thickness $d$ is, correspondingly,

$$
\phi_{s a m}(f)=\phi_{r e f}(f)+\frac{2 \pi(n(f)-1) f d}{c} .
$$

If the starting point of the sample scan is moved by a time $t_{\text {offset }}$ then the phase of the sample signal is corrected according to this,

$$
\phi_{\text {sam }}\left(f, t_{\text {offset }}\right)=\phi_{\text {ref }}(f)+\frac{2 \pi(n(f)-1) f d}{c}+2 \pi f t_{\text {offset }} \text {. }
$$

Thus, the phase difference between sample and reference is 


$$
\Delta \phi(f)=\phi_{\text {sam }}(f)-\phi_{r e f}(f)=\frac{2 \pi(n(f)-1) f d}{c}+2 \pi f t_{\text {offset }} .
$$

Based on the previous discussion about general phase unwrapping, the numerical problem is to unwrap the phase of the first term on the right-hand side (RHS) of Eq. (15). Since the second term (the offset between the two scans) adds to the phase difference between adjacent frequency points, it is most convenient to unwrap the phase difference without this delay term, and then add it after the phase jump correction.

Considering only the first term on the RHS of Eq. (15), for a frequency-dependent index of refraction the slope of the phase function is

$$
\frac{d(\Delta \phi(f))}{d f}=\frac{2 \pi d}{c}\left(f \frac{d n(f)}{d f}+n(f)-1\right) .
$$

Thus, if each discrete frequency is labeled $f_{k}=k \cdot \Delta f, k \in \mathbb{Z}$, the phase step between successive frequency points is

$$
\begin{aligned}
\delta\left(\Delta \phi\left(f_{k}\right)\right) & \approx \frac{2 \pi d \Delta f}{c}\left(f_{k} \frac{n\left(f_{k+1}\right)-n\left(f_{k}\right)}{\Delta f}+n\left(f_{k}\right)-1\right) \\
& =2 \pi \frac{d}{c T}\left(k\left(n\left(f_{k+1}\right)-n\left(f_{k}\right)\right)+n\left(f_{k}\right)-1\right) .
\end{aligned}
$$

For a nondispersive medium $(d n / d f=0)$ the above relation simplifies to

$$
\delta\left(\Delta \phi\left(f_{k}\right)\right)=2 \pi \frac{d}{c T}(n-1) .
$$

This shows an expected result: The longer the scan length $T$, the finer the frequency resolution is, and the smaller the phase step between successive frequency points. In this simplified situation, the required scan length $T$ that ensures that the absolute value of the phase difference between successive frequency points is smaller than $\pi$ is

$$
T>\frac{2 d}{c}|n-1|,
$$

which is identical to the difference in round-trip time in the sample and the air layer of the same thickness (the reference). In THz-TDS on thick samples, the scan time window is typically truncated right before the first round-trip echo of the sample, located at a time $2 n d / c$ after the first transmitted signal. This shows that while this truncation is acceptable in relation to the phase unwrapping, Eq. (19) gives the minimum time window required for correct unwrapping of the phase. If the sample is dispersive ( $d n / d f \neq 0)$, then the situation is a bit more complicated and slightly less favorable. In this case, the condition of absolute value of the phase step less than $\pi$ between successive frequency points can be written as

$$
T\left(f_{k}\right)>\frac{2 d}{c}\left|n\left(f_{k}\right)-1+k\left(n\left(f_{k+1}\right)-n\left(f_{k}\right)\right)\right| .
$$

Since the shape of the refractive index is typically not known in advance, this expression is difficult to use in the planning of an experiment. For this, Eq. (19) may still be a first, approximate guideline. 
These somewhat stringent conditions for the minimum length of the time window can be relaxed by the following consideration: The known phase slope difference [Eqs. (5)] can be seen as

$$
\Delta \phi_{0}=2 \pi f\left(t_{0, \text { sam }}-t_{0, r e f}\right) \equiv \frac{2 \pi(\bar{n}-1) f d}{c},
$$

where $\bar{n}$ is the frequency-averaged index of refraction of the sample. Thus, by subtracting this known phase difference, the new condition for excessive phase jumps is a modified version of Eq. (17), which now reads

$$
\delta\left(\Delta \phi^{\prime}\left(f_{k}\right)\right)=\frac{2 \pi d}{c T}\left(k\left(n\left(f_{k+1}\right)-n\left(f_{k}\right)\right)+n\left(f_{k}\right)-\bar{n}\right)
$$

where $\Delta \phi^{\prime}\left(f_{k}\right)=\Delta \phi\left(f_{k}\right)-\Delta \phi_{0}\left(f_{k}\right)$. Now the time window limitation (Eq. (20)) is modified to

$$
T\left(f_{k}\right)>\frac{2 d}{c}\left|n\left(f_{k}\right)-\bar{n}+k\left(n\left(f_{k+1}\right)-n\left(f_{k}\right)\right)\right| .
$$

Thus, unwrapping can be performed on $\Delta \phi^{\prime}$ and the offset $\Delta \phi_{0}$ then added back to the unwrapped phase difference. For nondispersive samples $(n(f) \approx \bar{n})$ there are then no practical limitations on the shortest time window to be used, at least with respect to correct phase unwrapping.

However, even the modified Eq. (22) in the case of dispersive media shows a feature that can be a complication for THzTDS measurements in two situations.

Firstly, TDS measurements with high bandwidth, for instance with experimental setups based on THz generation in twocolor air plasmas driven by femtosecond laser pulses [21-23] and THz detection by air-biased coherent detection (ABCD) [24], can reach frequencies of $30 \mathrm{THz}$ or more [25]. This is approximately an order of magnitude higher bandwidth than traditional THz-TDS, which is limited to a few THz upper frequency cut-off in practical measurements. The higher bandwidth in ABCD measurements requires a small time step $\Delta t$, and thus a large acquisition time. It may therefore be tempting to reduce the full scan length $T$ in such measurements, compared to a low-bandwidth THz-TDS measurement with larger time step. In such situations, one should still keep Eqs. (22) and (23) in mind.

Secondly, samples with large dispersion will show a significant variation of $n(f)$ so that it in general can deviate significantly from the average value of the refractive index determined from the arrival time of the pulses (Eq. (21)). Samples with sharp resonances will feature a fast-varying change in the refractive index at the resonance frequency. This can lead to a very large value of $\delta\left(\Delta \phi^{\prime}(f)\right)$ at the resonance frequency, and therefore the requirement of a unexpectedly large scan window. In practical terms, a sharp resonance leads to a long ringing after the main $\mathrm{THz}$ signal, and the requirement in Eq. (23) is equivalent to a recording of a sufficiently long time window that catches the full decay of the ringing signal (and therefore secures a significant frequency resolution to reproduce the phase variation across the resonance with sufficient fidelity). As can be seen from the discussion here, this time can easily exceed the time between subsequent round-trip echoes in the sample, and thus the selection of a suitable time window poses a fundamental problem. The manifestation of this resonance-related phase problem is a large phase jump across a resonant feature, giving rise to incorrect jump of the phase across that resonance. 
The implications of Eq. (23) with respect to both of these situations will be discussed after the following example of highbandwidth THz-TDS spectroscopic analysis.

\section{High bandwidth measurements of the refractive index of strongly dispersive materials}

To quantify the discussion above, let us consider another practical example. The material silicon carbide (SiC) is a highbandgap semiconductor with a strong transverse optic (TO) phonon at $24 \mathrm{THz}$. Intrinsic (undoped) SiC has high transparency in the $\mathrm{THz}$ region below the TO frequency, and has a strong phonon-induced dispersion of the refractive index across the $0-20 \mathrm{THz}$ region $[26,27]$. The dielectric function of $4 \mathrm{H} \mathrm{SiC}$ can be expressed as a sum of two Lorentz oscillator terms,

$$
\varepsilon(\omega)=\varepsilon_{\infty}+\frac{W_{T A}}{\omega_{T A}^{2}-\omega^{2}-i \omega / \tau_{T A}}+\frac{W_{T O}}{\omega_{T O}^{2}-\omega^{2}-i \omega / \tau_{T O}},
$$

where $\omega=2 \pi f$. For the $4 \mathrm{H}$ polytype, two terms contribute to the dielectric function, namely a very strong term ( $\left.W_{T O}\right)$ that describes the TO phonon, and a weak term $\left(W_{T A}\right)$ that describes a transverse acoustic (TA) phonon, which is infraredactive due to polytype-specific folding of the Brillouin zone [28]. The experiment and the results are described in detail in Ref. [28]. Important to note here is that the spectroscopy was performed with large-aperture samples of $\mathrm{SiC}(10 \mathrm{~cm}$ diameter) placed in the parallel section of the $\mathrm{THz}$ beam in the spectrometer, in order to obey the plane-wave approximation behind Eqs. (1) and (2) as closely as possible.

From the model dielectric function, the index of refraction (as well as extinction coefficient and absorption coefficient) can be found, $n+i \kappa=\sqrt{\varepsilon}, \alpha=2 \omega \kappa / c$. The permittivity, index of refraction, absorption coefficient, and associated phase shift after propagation through a $491-\mu \mathrm{m}$ slab of the material are shown in Figure 6.
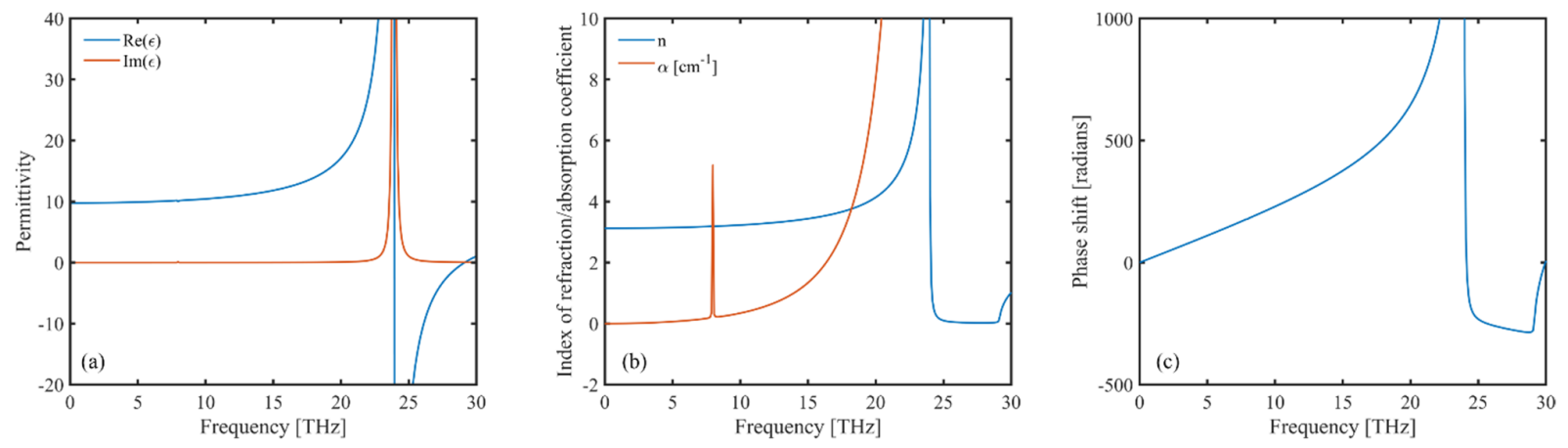

Figure 6: (a) Model permittivity based on Eq. (24), (b) index of refraction and absorption coefficient, and (c) associated phase shift through a $491-\mu \mathrm{m}$ thick slab of $4 \mathrm{H} \mathrm{SiC}$.

Raw time-domain recordings of an ultrabroadband $\mathrm{THz}$ transient transmitted through the sample are shown in Figure 7(a), and spectral amplitudes of the two signals are shown in Figure 7(b). The blue and red traces are the reference and sample signals, respectively. 

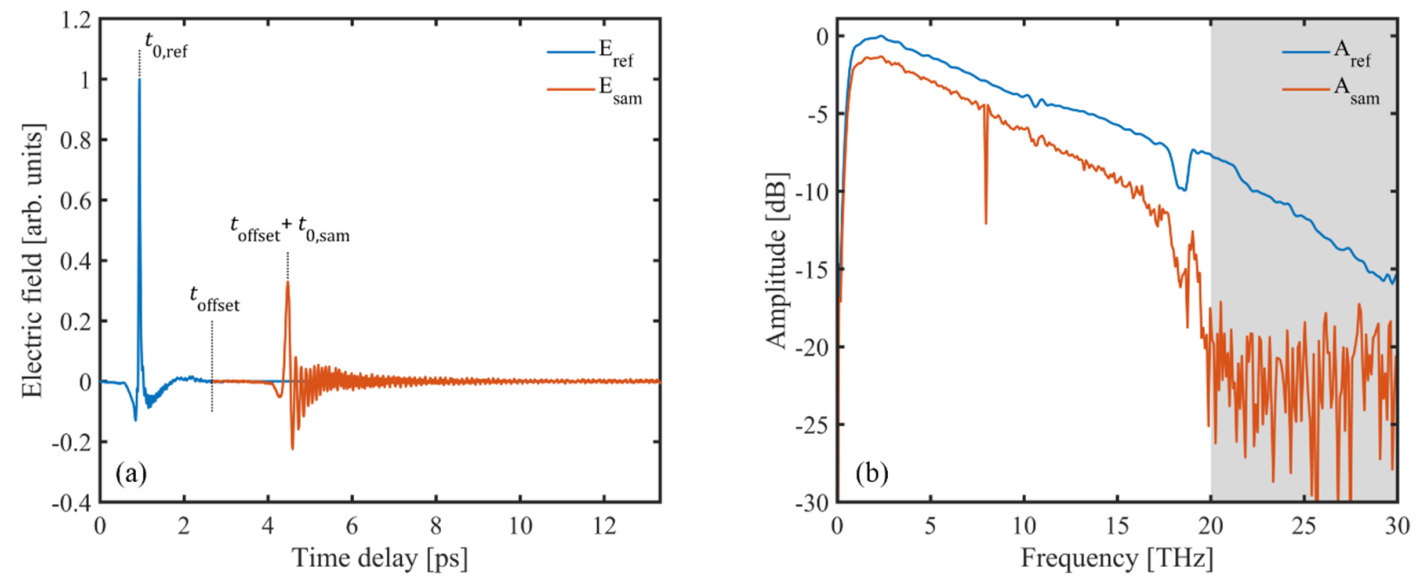

Figure 7: (a) THz-TDS data of transmission through a 491- $\mu \mathrm{m}$ thick $4 \mathrm{H} \mathrm{SiC}$ wafer (red trace) and the reference signal (blue). (b) Amplitude frequency spectra of the reference (blue) and sample (red) pulses.

The strong dispersion of the sample is evident from the extended ringing observed in the sample signal after the main pulse (red trace). Following the unwrapping procedures outlined in the previous discussion, we can find the phase of the reference and sample signals, as shown in Figure 8(a). The reference phase (blue curve) is, as expected, a linear function of frequency located precisely as predicted by the arrival time of the signal (gray dotted curve). The sample phase (red curve) follows the predicted phase (gray dashed curve) from the arrival time and the temporal offset between the scans at low frequencies, but then deviates significantly from this linear phase at high frequencies. An apparent shoulder in the sample phase is seen at $18 \mathrm{THz}$, before the sample signal is lost at frequencies above $20 \mathrm{THz}$ (see Figure 7(b)). The phase difference between sample and reference is plotted in Figure 8(b). The blue curve indicates the simple subtraction of the sample phase from the reference phase, and the green, dotted curve shows the model phase difference, based on the permittivity (Eq. (24)) and the sample thickness. Close to perfect agreement is seen up to $18 \mathrm{THz}$, after which a systematic deviation is seen.
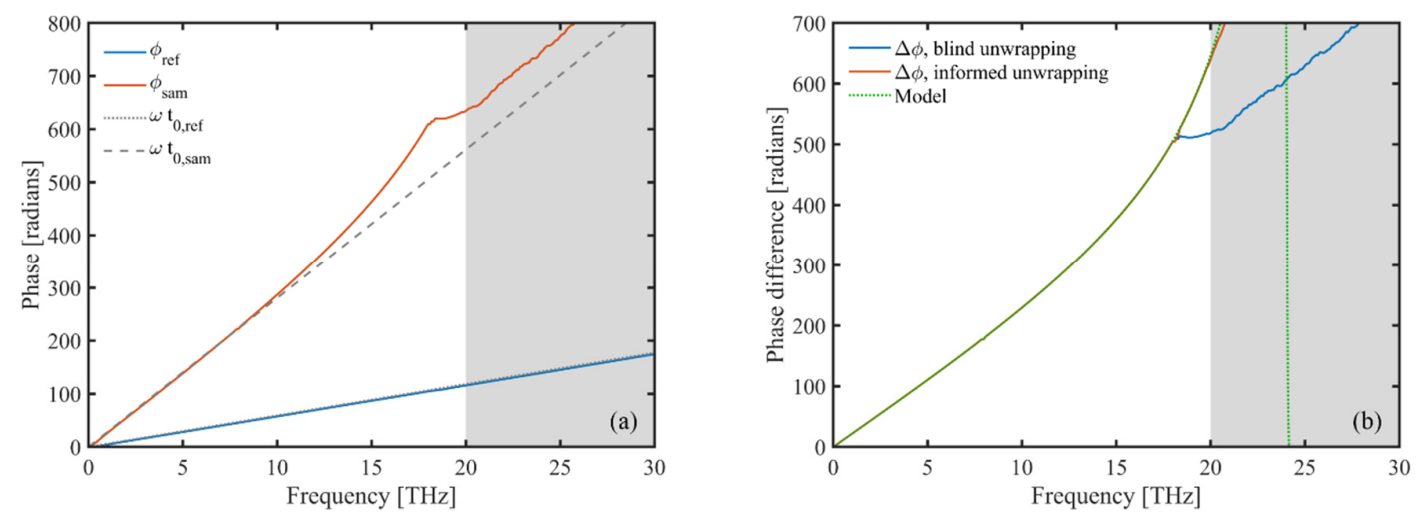

Figure 8: (a) Phases of the reference and sample signals in Figure 7, using optimal blind unwrapping. Notice the shoulder in the phase of the sample signal at approximately $18 \mathrm{THz}$. (b) Phase difference between sample and reference signals used for calculation of the refractive index. Dashed, green curve shows the model phase difference based on Eq. (24), blue curve is the blind unwrapped phase difference, and the red curve is the unwrapped phase difference, taking unphysical phase jumps into account. 
The deviation between the expected and unwrapped phase after $18 \mathrm{THz}$ is due to violation of the condition described by Eq. (23). The red curve in Figure 8(b) is a corrected version of the retrieved phase difference, as described in the following. Figure 9 plots the phase jump between adjacent frequency points of the unwrapped phase $\Delta \phi\left(f_{k}\right)-\Delta \phi\left(f_{k-1}\right)$ (red curve, calculated from the blue curve in Figure 8(b)) and that of the model phase data sampled with the same frequency resolution (blue curve, calculated from the green dotted curve in Figure 8). The green dashed line in Figure 9 indicates the $\pi$ threshold. It is clear that this threshold is crossed at $18 \mathrm{THz}$, and the unwrapping fails. Since the phase curve, from this point on, is offset by $2 \pi$ for every frequency point with respect to the previous one, the phase curve can be completely unwrapped by

$$
\Delta \phi_{\text {final }}\left(f_{k}\right)=\Delta \phi\left(f_{k}\right)+\left(k-k_{0}\right) \cdot 2 \pi
$$

where $k_{0}$ is the frequency index at which the correction starts. The resulting phase jump curve is shown in orange in Figure 9. With this additional optics-based unwrapping, the phase difference curve is now in full agreement with the model curve, as shown in Figure 8(b) (red curve).

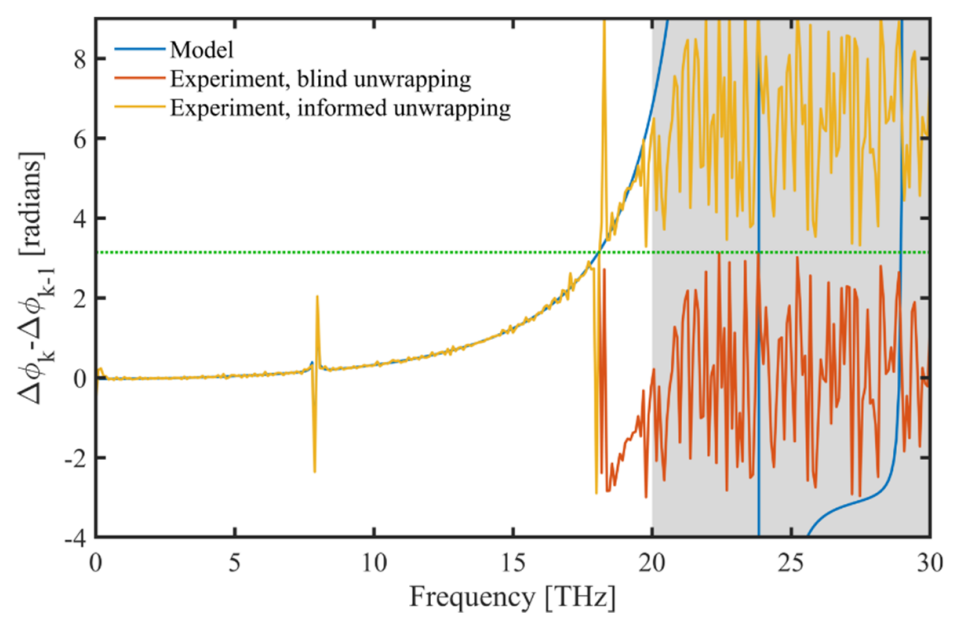

Figure 9: Phase jumps between adjacent frequency points.

With this final modification to the extracted phase difference between the sample and the reference signals, the index of refraction and the absorption coefficient can be calculated correctly, with results shown in Figure 10.
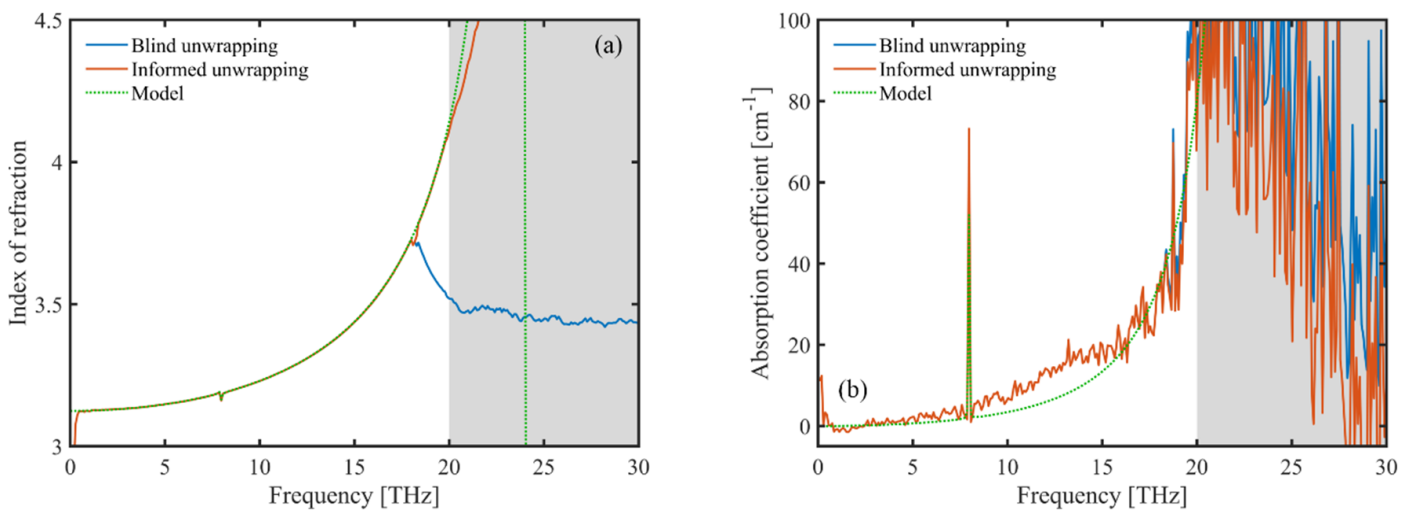

Figure 10: (a) Index of refraction and (b) absorption coefficient of $4 \mathrm{H} \mathrm{SiC}$ in the $0-20 \mathrm{THz}$ range. 
The correctly interpreted phase difference leads to a refractive index (Figure 10(a), red curve) in close agreement with the model (green dotted curve) for $4 \mathrm{H} \mathrm{SiC}$. In contrast, the original unwrapped phase difference leads to wrong behavior of the refractive index, with an apparent peak near $18 \mathrm{THz}$. Figure 10(b) shows the absorption coefficient, calculated by using the index of refraction estimated from the blind unwrapping (blue curve) and the informed unwrapping (red curve). In this specific example, the extracted absorption coefficient is not influenced significantly by the improved phase extraction, since the strong absorption dominates over reflection losses at the highest frequencies.

In general, we do not know the index of refraction of a new material before the measurement is actually performed. This makes general and fully automatic identification of such artifacts difficult. In these common situations, manual inspection of the phase jump curve (as illustrated in Figure 9) can be a documentable way to identify possible numerical problems with automated phase correction, followed by manual corrections. Another insight can be obtained by plotting the minimum time window required for a faithful representation of the phase (Eq. (23)). For the SiC spectroscopic data discussed above, the model dielectric function is again used, and the result is shown in Figure 11. Here the blue curve shows the frequency-dependent minimum scan length (Eq. (23)). Naturally, the scan length is a single value, so the use of this curve is to determine the highest value within the useful spectral range (here approximately 23 ps). However, as

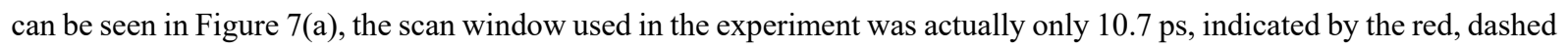
line in Figure 11. Two features are observed. Firstly, the very sharp phonon resonance at $8 \mathrm{THz}$ requires a time window of at least $8 \mathrm{ps}$ in order to be resolved without phase retrieval problems with the chosen frequency resolution. This is fulfilled by the experiment. Secondly, it can then be seen that for frequencies above approximately $18 \mathrm{THz}$, the experiment does not sample the transmitted THz transient over a sufficiently large temporal window. Notice that this frequency is the same as the critical frequency for excessive phase jumps observed in Figure 9(a). In practice, the useful scan window length is limited by the signal-to-noise ratio obtainable in the experiment. At the high frequencies in question here, the signal strength is very low, and approaching the noise floor of the experiment (see Figure 7(b)). If the temporally dispersed signal at late times from the sample has a smaller amplitude than the noise of the experiment, there is no advantage of sampling the waveform, and the selected scan window becomes a compromise between the ideal length and accumulation of additional broadband noise. 


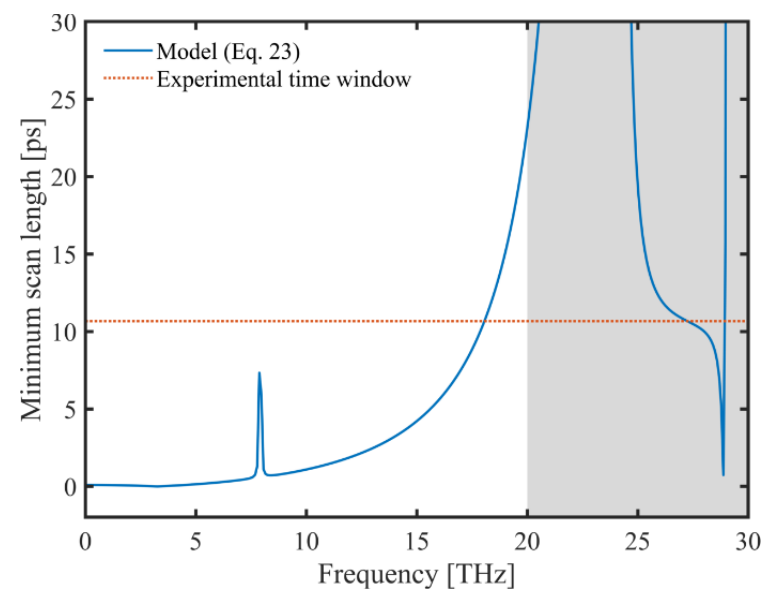

Figure 11. Plot of the minimum time window to be used when recording THz-TDS data of the SiC sample discussed in the text. The blue curve is calculated with Eq. (23), using the model dielectric function for SiC (Eq. (24)). The red, dashed line indicates the scan window used in the experiment (Figure 7(a)).

\section{Conclusions}

To summarize, this tutorial has discussed the most important pitfalls to consider when analyzing the phase of the optical signals in THz time-domain spectroscopy. Six steps, summarized by Eqs. (9)-(12), form a stable method for phase retrieval that will work in virtually all situations commonly encountered in THz-TDS. These steps can be implemented in a rather straightforward manner in data analysis software, and requires no user interaction. The seventh step (summarized by Eq. (25)), to be employed if the sample material is strongly dispersive, typically requires inspection and decisions by the user, but represents a documentable additional treatment of the phase in such situations, in order to extract as much consistent information from a given experiment as possible.

\section{References}

[1] M. van Exter, C. Fattinger, and D. Grischkowsky, "Terahertz time-domain spectroscopy of water vapor," Opt. Lett. 14, 1128-1130 (1989).

[2] W. Withayachumnankul, and M. Naftaly, "Fundamentals of Measurement in Terahertz Time-Domain Spectroscopy," J. Infrared Millim. Terahertz Waves 35, 610-637 (2014).

[3] M. Naftaly, "An international intercomparison of THz time-domain spectrometers," in 201641 st International Conference on Infrared, Millimeter, and Terahertz waves (IRMMW-THz)(2016), p. 7758763.

[4] L. Duvillaret, F. Garet, and J. L. Coutaz, "A reliable method for extraction of material parameters in terahertz timedomain spectroscopy," IEEE J. Sel. Top. Quantum Electron. 2, 739-746 (1996).

[5] L. Duvillaret, F. Garet, and J. L. Coutaz, "Highly precise determination of optical constants and sample thickness in terahertz time-domain spectroscopy," Appl. Opt. 38, 409-415 (1999).

[6] I. Pupeza, R. Wilk, and M. Koch, "Highly accurate optical material parameter determination with THz time-domain spectroscopy," Opt. Express 15, 4335-4350 (2007).

[7] M. Scheller, C. Jansen, and M. Koch, "Analyzing sub-100- $\mu \mathrm{m}$ samples with transmission terahertz time domain spectroscopy," Opt. Comm. 282, 1304-1306 (2009).

[8] M. Kruger, S. Funkner, E. Brundermann, and M. Havenith, "Uncertainty and Ambiguity in Terahertz Parameter Extraction and Data Analysis," J. Infrared Millim. Terahertz Waves 32, 699-715 (2011).

[9] M. Naftaly, and R. Dudley, "Methodologies for determining the dynamic ranges and signal-to-noise ratios of terahertz time-domain spectrometers," Opt. Lett. 34, 1213-1215 (2009).

[10] P. U. Jepsen, and B. M. Fischer, "Dynamic range in terahertz time-domain transmission and reflection spectroscopy," Opt. Lett. 30, 29-31 (2005). 
[11] T. J. Parker, "Dispersive Fourier transform spectroscopy," Contemporary Physics 31, 335-353 (1990).

[12] J. E. Chamberlain, J. E. Gibbs, and H. A. Gebbie, "Refractometry in the Far Infra-red using a Two-beam Interferometer," Nature 198, 874 (1963).

[13] D. Grischkowsky, S. Keiding, M. van Exter, and C. Fattinger, "Far-infrared time-domain spectroscopy with terahertz beams of dielectrics and semiconductors," J. Opt. Soc. Am. B 7, 2006-2015 (1990).

[14] N. M. Israelsen, C. R. Petersen, A. Barh, D. Jain, M. Jensen, G. Hannesschläger, P. Tidemand-Lichtenberg, C. Pedersen, A. Podoleanu, and O. Bang, "Real-time High-Resolution Mid-infrared Optical Coherence Tomography," arXiv:1810.05445 [physics.optics] (2019).

[15] C. L. Koch-Dandolo, T. Filtenborg, K. Fukunaga, J. Skou-Hansen, and P. U. Jepsen, "Reflection terahertz timedomain imaging for analysis of an 18th century neoclassical easel painting," Appl. Opt. 54, 5123 (2015).

[16] C. L. K. Dandolo, and P. U. Jepsen, "Wall Painting Investigation by Means of Non-invasive Terahertz Time-Domain Imaging (THz-TDI): Inspection of Subsurface Structures Buried in Historical Plasters," J. Infrared Millim. Terahertz Waves 37, 198-208 (2015).

[17] A. J. Huber, F. Keilmann, J. Wittborn, J. Aizpurua, and R. Hillenbrand, "Terahertz Near-Field Nanoscopy of Mobile Carriers in Single Semiconductor Nanodevices," Nano Lett. 8, 3766-3770 (2008).

[18] M. Eisele, T. L. Cocker, M. A. Huber, M. Plankl, L. Viti, D. Ercolani, L. Sorba, M. S. Vitiello, and R. Huber, "Ultrafast multi-terahertz nano-spectroscopy with sub-cycle temporal resolution," Nat. Photon. 8, 841 (2014).

[19] P. Kužel, H. Němec, F. Kadlec, and C. Kadlec, "Gouy shift correction for highly accurate refractive index retrieval in time-domain terahertz spectroscopy," Opt. Express 18, 15338-15348 (2010).

[20] P. U. Jepsen, D. G. Cooke, and M. Koch, "Terahertz spectroscopy and imaging - Modern techniques and applications," Laser Photon. Rev. 5, 124-166 (2011).

[21] D. J. Cook, and R. M. Hochstrasser, "Intense terahertz pulses by four-wave rectification in air," Opt. Lett. 25, 1210$1212(2000)$

[22] M. Kress, T. Löffler, S. Eden, M. Thomson, and H. G. Roskos, "Terahertz-pulse generation by photoionization of air with laser pulses composed of both fundamental and second-harmonic waves," Opt. Lett. 29, 1120-1122 (2004).

[23] X. Xie, J. M. Dai, and X. C. Zhang, "Coherent control of THz wave generation in ambient air," Phys. Rev. Lett. 96, $075005(2006)$

[24] J. Dai, X. Xie, and X. C. Zhang, "Detection of broadband terahertz waves with a laser-induced plasma in gases," Phys. Rev. Lett. 97, 103903 (2006).

[25] T. Wang, P. Klarskov, and P. U. Jepsen, "Ultrabroadband THz Time-Domain Spectroscopy of a Free-Flowing Water Film," IEEE Trans. Terahertz Sci. Technol. 4, 425-431 (2014).

[26] M. Naftaly, J. F. Molloy, B. Magnusson, Y. M. Andreev, and G. V. Lanskii, "Silicon carbide - a high-transparency nonlinear material for THz applications," Opt. Express 24, 2590-2595 (2016).

[27] M. P. Fischer, J. Bühler, G. Fitzky, T. Kurihara, S. Eggert, A. Leitenstorfer, and D. Brida, "Coherent field transients below $15 \mathrm{THz}$ from phase-matched difference frequency generation in 4H-SiC," Opt. Lett. 42, 2687-2690 (2017).

[28] A. T. Tarekegne, B. Zhou, K. Kaltenecker, K. Iwaszczuk, S. Clark, and P. U. Jepsen, "Terahertz time-domain spectroscopy of zone-folded acoustic phonons in 4H and 6H silicon carbide," Opt. Express 27, 3618-3628 (2019). 